\title{
Study on the Efficacy Evaluation of Oral Sequential Tamoxifen in Postmenopausal Women with Hormone-dependent Breast Cancer
}

\author{
Na Li, Yahua Sun, Sheng Zhao, Haiou Liu, Kun Zhao
}

The Third Affiliated Hospital of Qiqihar Medical University, Qiqihar, Heilongjiang, 161000

Keywords: Sequential tamoxifen tablets; breast cancer; efficacy

\begin{abstract}
Third Affiliated Hospital of Qiqihar Medical College since 2013. Inclusion criteria for patients with early surgical resection and complete pathology. There was complete immunohistochemical data, and the immunohistochemical SP method confirmed ER positive and/or PR positive. All the 135 patients in this group were female, aged 47-75 years old, with an average of 57.6 years old. All of them were stage II breast cancer patients, and all underwent radical mastectomy. All of them were confirmed to be estrogen receptor positive by immunohistochemistry. All are women. All the hormones were verified in the menopause. After grouping, the treatment group 1 was treated with $2.5 \mathrm{mg}$ of letrozole. After one year of treatment, the patient was switched to oral tamoxifen $10 \mathrm{mg}$ twice a day. Treatment group 2 oral administration of letrozole $2.5 \mathrm{mg}$, once a day treatment, every three months. RESULTS: The 5-year local recurrence rate was $13.8 \%$ in the treatment group 1 and the distant metastasis rate was $8.2 \%$. The treatment group 2 was $10.4 \%$ and $10.4 \%$, respectively. There was no significant difference in efficacy between treatment group 1 and treatment group 2.
\end{abstract}

\section{Introduction}

Breast cancer is one of the most common female malignant tumors, which seriously affects women's health and living conditions. About 2/3 of breast cancer patients' cancer tissues are estrogen-dependent. Therefore, reducing the stimulation of estrogen on tumor cells can effectively control The disease continues to develop, and the sensitivity of tumor cells to estrogen and the reduction of estrogen levels in the body determine the efficacy of endocrine therapy. Although the incidence of breast cancer is harmful to women's health and the incidence is increasing, the application of endocrine therapy has increased its effective treatment, and it has become one of the best solid tumors [1]. With the emergence of anti-estrogen drugs such as tamoxifen and aromatase inhibitors such as letrozole, endocrine therapy has made new progress in reducing mortality and recurrence rates and improving survival rates in breast cancer patients [2]. Tamoxifen has been used in endocrine therapy for more than 30 years. Its structure is similar to that of estrogen. It competes with estrogen receptor (ER) and inhibits the action of estrogen [3]. Tamoxifen tablets are A non-street estrogen drug, which is a component of phenoxyamine, is used to treat breast cancer, advanced ovarian cancer and some benign gynecological diseases, and is one of the anti-tumor drugs with less side effects. The author reviewed and analyzed the efficacy of tamoxifen citrate tablets in the treatment of breast cancer in our hospital since 2013. The results are reported as follows:

\section{Source of information}

135 breast cancer patients admitted to the Third Affiliated Hospital of Qiqihar Medical College since 2013. Inclusion criteria for patients with early surgical resection and complete pathology. There was complete immunohistochemical data, and the immunohistochemical SP method confirmed ER positive and/or PR positive. All the 135 patients in this group were female, aged 47-75 years old, with an average of 57.6 years old. All patients were stage II breast cancer patients, and all underwent radical mastectomy. All cases were confirmed by estrogen receptor immunohistochemistry and lymph node metastasis $\geq 1$ piece, tumor $\geq 5 \mathrm{~cm}$. All are women. The 
hormone hexa-chemical verification was in the menopause period. After grouping, one group received $2.5 \mathrm{mg}$ of letrozole. After 2 days of treatment, the patient was switched to oral tamoxifen $10 \mathrm{mg}$ twice a day. Another group of oral letrozole $2.5 \mathrm{mg}$, once a day treatment, surgical medical examination, if the surgical examination of new nodules, need to be confirmed by pathology, every three months to review liver and gallbladder pancreatic spleen ultrasound, uterine attachment color Doppler ultrasound, breast color ultrasound, lymph nodes Color Doppler, chest CT, review CEA, CA-153, CA-125 every six months. Check bone ECT every year, head MRI. One of the above positive conclusions was judged to be resistant to endocrine therapy, and the SPSS 17.0 statistical software package was used for statistical analysis of the data (rank sum test of group design) to clarify that the two methods have the control of breast cancer recurrence and metastasis. There was no significant difference.

\section{Results}

According to the American Cancer Joint Committee (AJCC) breast cancer TNM staging, 46 patients (34\%) with clinical stage I in the letrozole monotherapy group and 46 patients (34\%) with stage II patients, tamoxifen There were 22 patients (16.1\%) in stage I and 21 patients (15.6\%) in stage II. There was no significant difference between the two groups, $\mathrm{P}>0.05$, see Table 1 .

Table 1 Comparison of clinical stage between the two groups of patients

\begin{tabular}{cccc}
\hline \multirow{2}{*}{ Clinical stage } & \multicolumn{2}{c}{ Gr } & \multirow{2}{*}{ Total } \\
& He Moxi powder group Letrozole group & (Case \%) \\
& Case \%) & Case \%) & $92(68.2 \%)$ \\
I & $46(34.1 \%)$ & $46(34.1 \%)$ & $43(31.8 \%)$ \\
\hline II & $22(16.3 \%)$ & $21(15.5 \%)$ & $135(100 \%)$ \\
\hline
\end{tabular}

Positive cells with brown-yellow particles appeared in the nucleus of each of the 10 high-power fields randomly selected. The positive cells were $<14 \%$ low expression and the positive cells were $\geq 14 \%$ high expression. Among the tamoxifen sequential groups, 38 patients $(55.9 \%)$ had low expression, and 30 patients (44\%) had high expression. The low expression of Ki-67 in the letrozole monotherapy group was 35 (52.2\%), and the high expression was 32 (23.7\%). There was no significant difference between the two groups, $\mathrm{P}=0.501$, see Table 2 .

Table 2 Comparison of Ki-67 expression between the two groups

\begin{tabular}{cccc}
\hline \multirow{2}{*}{ Ki-67 } & \multicolumn{2}{c}{ Gr. } & Total \\
& Hemoxi powder group, letrozole group & \\
\hline Low expression & 38 & 30 & 68 \\
High expression & 35 & 32 & 67 \\
\hline Total & 73 & 62 & 135 \\
\hline
\end{tabular}

The patient was admitted to the hospital every 3 months after the medication, and the whole chest X-ray, abdominal B-ultrasound and whole body bone isotope scans were performed for half a year. Local recurrence includes local tumor recurrence and ipsilateral or contralateral axillary and paraclavicular lymph node metastasis. Distal metastasis includes metastasis of lung, liver, stomach, etc., and formation of malignant thoracic and ascites. The 5-year local recurrence rate and the proportion of distant metastasis in the treatment group were not significantly different from those in the control group $(\mathrm{P}<0.05)$. The results are shown in Table 3. 
Table 3 Observation of 5-year postoperative indexes in two groups of patients

\begin{tabular}{clcc}
\hline Clinical stage & $\begin{array}{l}\text { Local } \\
\text { Case (\%) }\end{array}$ & $\begin{array}{c}\text { recurrence } \\
\text { Case (\%) }\end{array}$ & Case \\
\hline Gr. 1 & $8(13.2 \%)$ & $6(8.8 \%)$ & 68 \\
Gr. 2 & $7(10.4 \%)$ & $7(10.4 \%)$ & 67 \\
\hline
\end{tabular}

\section{Discussion}

Clinically, breast cancer, in addition to surgical treatment, emphasizes comprehensive treatment. The biological behavior of breast cancer is related to endocrine disorders, and endocrine therapy for breast cancer has received more and more attention, and it has become another important treatment method other than surgical treatment [4]. Many scholars have reported that estrogen may cause breast cancer. Overexpression of estrogen receptors in breast cancer specimens may support this theory. As a non-steroidal estrogen drug, tamoxifen tablets can compete with estrogen in the body for receptors of target cells, inhibit cell division and hyperproliferation, and thus inhibit tumorigenesis.

Breast cancer is a systemic disease, and endocrine therapy plays an important role in systemic comprehensive treatment of hormone-responsive breast cancer. Endocrine therapy has fewer side effects, does not affect the patient's quality of life, and has good tolerance [5]. The most commonly used endocrine therapy drugs for breast cancer: anti-estrogens, progesterones, aromatase inhibitors and LH-RH analogues [6]. Tamoxifen has been used as an anti-estrogen representative drug for endocrine therapy for more than 30 years. The standard drug for endocrine therapy has remarkable curative effect and less side effects. A 2005 meta-analysis of EBCTCG showed that the risk of breast cancer death in women under 50 and 50-69 years of age was 57\% and $45 \%$, respectively, after chemotherapy with tamoxifen for 5 years [7]. However, with the development and application of aromatase inhibitors, more and more clinical data indicate that the third-generation aromatase inhibitors have superior efficacy and better tolerance than tamoxifen. Tamoxifen can be used for pre-menopausal or post-menopausal hormone receptor-positive patients due to different mechanisms of action. Letrozole is a highly specific non-steroidal third-generation aromatase inhibitor mainly used for menopause. After hormone-responsive patients. In a number of international, multicenter clinical randomized trials, single letrozole showed superior status in the treatment of early postmenopausal breast cancer patients compared with tamoxifen alone. Mouridsen [8] conducted a trial of 025 in the future to compare the efficacy of tromethamine and tamoxifen in the treatment of advanced breast cancer. The 907 patients enrolled in the study were postmenopausal, ER-positive patients with advanced breast cancer, with a median follow-up of 18 After a month, the results showed that the disease progression time (TTP) of letrozole and tamoxifen was 9.4 months and 6 months, respectively; the objective response rates of letrozole and tamoxifen were $30 \%$ and $20 \%$, The difference was significant $(\mathrm{P}<0.05)$. This study not only provides guidance for clinicians on safe and effective drug use, but also provides a choice of reasonable endocrine therapy options that combine specific conditions such as self-tolerance, drug resistance, perimenopause, and economic status.

Endocrine therapy plays an important role in the treatment of patients with hormone-responsive breast cancer. Tamoxifen and letrozole are two endocrine therapy drugs with different mechanisms of action. This article compares the efficacy and safety of sequential temoxime treatment for 2 to 3 years and letrozole monotherapy for 5 years after tamoxifen treatment. The tamoxifen sequential letrozole group There was no significant difference in the 5-year disease-free survival rate between the letrozole group. Patients with postmenopausal early hormone-responsive breast cancer who have a relatively low risk of relapse may consider tamoxifen sequential letrozole monotherapy, and patients with breast cancer who are at relatively high risk of relapse may consider letrozole monotherapy Program. This topic is based on solving the difficulties encountered in clinical practice, clear postmenopausal breast cancer ER, PR receptor positive oral letrozole 2 years later changed to tamoxifen 3 years endocrine therapy and oral oral letrozole treatment 5 There is no 
difference in the efficacy of the year. The study of this topic provides data support for breast cancer patients who cannot tolerate aromatase inhibitors after hormone receptor-positive menopause. Breast cancer is one of the common malignant tumors in women, and the incidence has continued to increase in recent years. It is the first female malignant tumor. Although there are many treatments for breast cancer, such as surgery, radiation therapy, chemotherapy, targeted therapy, immunotherapy, and endocrine therapy. Because of the long survival time of breast cancer patients, local recurrence and distant metastasis are prone to occur during the period. Postmenopausal breast cancer patients adhere to endocrine therapy, which directly affects the treatment and prognosis of patients. The research results of this project can be widely used in clinical practice and have strong practicability. It can solve the problem of oral letrozole endocrine therapy intolerance in postmenopausal women with ER and PR receptor positive breast cancer. The implementation of the project can greatly improve the research capacity, scientific research level and medical technology level of our hospital. It can also promote the development of related departments such as pathology, laboratory, functional, imaging, oncology, etc., attracting more patients to visit our hospital. .

\section{Acknowledgements}

Fund Project: Qiqihar Science and Technology Program: Evaluation of the efficacy of oral aromatase inhibitor sequential tamoxifen in postmenopausal hormone-dependent breast cancer patients. (Item No.: SFZD-2017018)

\section{References}

[1] Jacobs JR, Bovasso GB. Early and chronic stress and their relation to breast cancer [J]. Psychol Med, 2000, 30(3): 669-678.

[2] Robortson JFR, Nicholson RI, Hayes DF. Endocrine therapy of breast cancer [M]. London: Martin Dunitz, 2002: 45-62.

[3] Obiorah 1, Jordan V C. Progress in endocrine approaches to the treatment and prevention of breast cancer [J]. Maturitas, 2011, 70(4): 315-321.

[4] Li Dianling. Review and prospect of breast cancer prevention and treatment research [J]. Foreign Medical Oncology, 2002, 2 (1): 14

[5] $\mathrm{Xu}$ Binghe. Current status and prospects of endocrine therapy in breast cancer [J]. Chinese Journal of Tumor Prevention and Treatment, 2006, 13:161-164.

[6] Zhang Honggang, Sun Yan, Li Erfan. Multi-center clinical study of combined chemotherapy and radiotherapy for malignant tumors [J]. Chinese Journal of Clinical Oncology, 2001, 28 (4): 282-287.

[7] Early Breast Cancer Trialists' Collaborative Group (EBCTCG) Davies C, Godwin J, et al. Release of breast cancer hormone receptors and other factors to the efficacy of adjuvant tamoxifen: patient-level meta-analysis of randomized trials [J] .Lancet, 2011, 378 (9793): 771-784.

[8] Mouridsen H, Gershanovich M, Sun Y, et al. Superior-efficacy of letrozole versus tamoxifen as first-line therapy for postmenopausal women with advanced breast 